\title{
Análisis de competencias en el Grado de Ingeniería Forestal para su adaptación al Espacio Europeo de Educación Superior
}

\author{
Esperanza Ayuga-Téllez ${ }^{(1)}$, Concepción González-García ${ }^{(1)}$ y María A. Grande-Ortiz ${ }^{(2)}$ \\ Universidad Politécnica de Madrid, ETSI Montes, (1) Departamento de Economía y Gestión \\ Forestal, \\ (2) Departamento de Física y Mecánica Fundamentales y Aplicadas a la Ingeniería Agroforestal. \\ Ciudad Universitaria s/n, 28040. Madrid-España (e-mail: esperanza.ayuga@upm.es; \\ concepción.gonzalez@upm.es; m.angeles.grande@upm.es)
}

Recibido Jun. 03, 2010; Aceptado Jul. 06, 2009; Versión final recibida Jul. 20, 2010

\section{Resumen}

En este trabajo se establecen las competencias genéricas y específicas a conseguir en titulaciones de Ingeniería Forestal adaptadas al Espacio Europeo de Educación Superior en España. Basándose en el concepto de Ingeniería Forestal, la legislación y las recomendaciones de asociaciones de ingenieros europeos y latino americanos, se seleccionaron diez competencias genéricas y catorce específicas. Los resultados se validaron mediante la técnica de investigación cualitativa de grupos naturales de discusión. Las materias para desarrollar estas competencias se agruparon en básicas, fundamentos de la ingeniería y ciencias agroforestales. El estudio muestra que las ciencias agroforestales requieren formación práctica para adquirir competencias específicas de la Ingeniería Forestal.

Palabras clave: EEES; competencias; proyecto docente; educación superior; ingeniero forestal

\section{Analysis of skills in the Degree in Forestry Engineering for its adaptation to the European Higher Education Space}

\begin{abstract}
In this work generic and specific skills that students of Forest Engineering degrees must obtain in the framework of the European Higher Education Space in Spain are established. Based on the concept of Forest Engineering, Spanish legislation and the recommendations of associations of European and Latin American engineers, ten generic and fourteen specific skills were selected. The results were validated by qualitative research technique of natural group discussion. The materials to develop these skills were grouped into basic, fundamentals of engineering and agroforestry science. The study shows that agroforestry sciences require practical training to acquire specific skills in Forest Engineering.
\end{abstract}

Keywords: EHEA; skills; teaching project; higher education; forest engineer 


\section{INTRODUCCIÓN}

En la actualidad, el avance en los transportes y las comunicaciones han conseguido acercar el mundo entero. Estamos en un mundo globalizado, donde el acceso a la información en cualquier instante y en cualquier punto del globo terrestre es casi una realidad (Ayuga, 2008). La movilidad de los trabajadores y el acceso a gran cantidad de información existente, mediante los medios informáticos desarrollados, revolucionan el panorama en que los profesionales desarrollan su actividad (BBVA, 2008; Korsunsky, 2010). Ante esta perspectiva de cambios y de enormes posibilidades, el profesional que quiera tener acceso a todas las oportunidades que se le ofrecen actualmente debe estar preparado para entender esos cambios y adaptarse a ellos rápidamente (Committee on the Offshoring of Engineering, 2008; Mardam-Bey et al, 2008). En la Comunidad Económica Europea, la normativa existente sobre movilidad de profesionales y la necesidad de aumentar su competitividad fomentan la preocupación sobre la formación adecuada de profesionales europeos. Así, con el EEES, se pretende conseguir una "Europa del conocimiento" en la que los sistemas europeos educativos se conviertan en una referencia de calidad mundial. En particular, se trata de promover el desarrollo curricular, la cooperación institucional, esquemas de movilidad y programas integrados de estudios, de formación y de investigación (Fredriksson, 2003). El concepto de ingeniero también ha ido cambiando con el tiempo. Hoy la sociedad considera ingenieros a personas que realizan tareas impensables hace cien años. Algunos ejemplos son la ingeniería genética, la ambiental, la microelectrónica o la aeroespacial. De esta diversificación de tareas se concluye que el concepto de ingeniero es cada día más general y abstracto, para poder admitir en su tronco a todas esas nuevas ramas (Tomisawa, 2006).

Las diversas ramas de la Ingeniería tienen distintos orígenes y objetivos particulares, presentan un grado distinto de contacto con la naturaleza y sus leyes, y han nacido en épocas muy distintas. Pero todas comparten lo esencial: el espíritu aplicado (práctico), los tipos de tareas, la complejidad de los problemas y la forma sistemática de resolverlos, la fiabilidad y rigor de los modelos manejados, y la exigencia de calidad en los resultados (McLeod, 2010). La Ingeniería Forestal (también denominada en España como Ingeniería de Montes) es una disciplina que tuvo sus inicios en Alemania (en 1811 se fundó la primera escuela forestal), pretendiendo cubrir la necesidad de mejorar la productividad de los montes. Con el transcurso del tiempo, la profesión se fue perfilando hacia el desarrollo y optimización del ámbito forestal: selvicultura, aprovechamiento, elaboración y transformación de productos forestales, infraestructuras específicas, etc. El Ingeniero Forestal, por tanto, tiene su acción profesional en el monte, concepto que abarca un extenso territorio y que corresponde a todo lo que supone una Naturaleza menos transformada por la actividad humana y que es origen de recursos y bienes absolutamente imprescindibles para el hombre actual. La preocupación actual por el cuidado y sostenibilidad de los recursos naturales ha propiciado que la sociedad demande profesionales que, por encima de la obtención de beneficios del Monte, realice tareas de protección y conservación del Medio Ambiente. En este contexto, y aprovechando la oportunidad que brinda la adaptación de titulaciones al EEES, se han revisado y analizado las competencias genéricas y específicas para el ingeniero forestal, tanto a nivel europeo como iberoamericano. Se organizaron grupos de discusión con expertos en materia de ingeniería agro-forestal. Del consenso de las reuniones y la información reunida, se presentan agrupadas las competencias que se consideran necesarias para un plan de estudios de grado en ingeniería forestal.

\section{ANÁLISIS DE DOCUMENTACIÓN Y METODOLOGÍA}

Para obtener los resultados de este trabajo se han integrado diferentes materiales: los trabajos realizados para la homogeneidad de formación en el Espacio Europeo de Educación Superior; y los relacionados con las competencias que se requieren en el ejercicio profesional de cualquier rama de la ingeniería, junto con las del ingeniero forestal.

\section{El Espacio Europeo de Educación Superior}

En la última década del siglo XX se manifiesta en Europa la preocupación por unificar la formación de profesionales de la zona Comunitaria, debido a las normas existentes sobre movilidad de 
estudiantes y profesionales. Esta preocupación se plasma en la "Declaración de Bolonia", documento en el que los ministros de 29 países se comprometían a conseguir en el año 2010 un Espacio Europeo de Educación Superior (EEES), para lo cual, formularon seis líneas de actuación:

1. Adopción de un sistema legible y comparable de títulos mediante la incorporación del "Suplemento al Diploma", Diploma Supplement.

2. Adopción de un sistema basado, esencialmente, en dos ciclos. El acceso al segundo ciclo requerirá haber superado totalmente el primer ciclo, con una duración mínima de tres años.

3. Establecimiento de un sistema de créditos European Credit Transfer System (ECTS), centrados en el trabajo y el aprendizaje del estudiante.

4. Promoción de la movilidad.

5. Promoción de la cooperación europea para asegurar la calidad, mediante el desarrollo de criterios y metodologías educativas comparables.

6. Promoción de la dimensión europea de la educación superior.

Posteriormente, se han incorporado nuevos países y se han realizado reuniones cada dos años, en Praga (2001), en Berlín (2003), en Bergen (2005), en Londres (2007) y en Benelux (2009). En dichas reuniones se hizo balance de los progresos realizados y se establecieron las directrices para la continuación del proceso. En concreto, se ha resaltado la importancia de la formación continua, la empleabilidad y la dimensión social de dicha formación (Universia, 2009). España es uno de los países que firmaron la declaración de Bolonia y por tanto uno de los que, desde el comienzo, estudia cómo armonizar la educación impartida en sus Universidades con los principios desarrollados en dichas reuniones. En esta línea, las Universidades de Deusto (España) y Groningen (Holanda) colaboraron en un proyecto piloto denominado Proyecto "Tuning - Sintonizar las estructuras educativas de Europa" (http://tuning.unideusto.org/tuningeu).

\section{Formación en competencias}

El proyecto "Tuning" se caracteriza por cuatro grandes ejes de actuación, que cuentan con la colaboración de varios equipos de expertos: 1) competencias genéricas, 2) competencias disciplinarias específicas, 3) el papel del sistema ECTS como sistema de acumulación, y 4) la función del aprendizaje, la docencia, la evaluación y el rendimiento en relación con el aseguramiento y la evaluación de la calidad. Así, según el proyecto Tuning "una competencia o conjunto de competencias es aquella en que una persona pone en práctica determinada capacidad o habilidad para desarrollar una labor haciéndola de tal forma que se pueda evaluar su consecución." En el desarrollo de este proyecto, se comienza por identificar, como puntos de referencia para elaborar programas de estudios comparables e intercambiables, los resultados del aprendizaje y las competencias. En uno de sus primeros trabajos se concretan, a escala europea, las competencias genéricas y específicas para cada disciplina, en una serie de campos temáticos: Matemáticas, Geología, Empresariales, Enfermería, Estudios Europeos, Historia, Ciencias de la Educación, Física y Química (Tuning Project, 2003). Los ECTS y su evaluación en relación con los resultados del aprendizaje (Tuning Project, 2006), así como el papel de sus ejes de actuación en el diseño de programas de educación superior europeos son los resultados más recientes del proyecto (Tuning Project, 2008).

La formación en competencias es, por tanto, uno de los objetivos principales que deben abordarse en las titulaciones de grado adaptadas al EEES. El profesor de Miguel (2004 y 2006) define las competencias como "una combinación compleja de conocimientos, técnicas, habilidades y valores que posibilita desarrollar adecuadamente una función, tarea o actividad en el ámbito profesional. Cabe distinguir entre el conjunto de conocimientos ("saber"), habilidades ("saber hacer") y actitudes ("saber estar")". Por su generalidad se distinguen tres tipos de competencias: a) genéricas, aquellas que son necesarias como base para el desempeño en cualquier área ocupacional, b) específicas, son las que se requieren para el desempeño de una función específica, dentro de un área o sector ocupacional, c) transversales, las que se requieren en diversas áreas ocupacionales o que son transferibles entre distintas actividades de un sector $u$ organización (McEvoy et al, 2005; Deakin, 2008; Kallioinen, 2010). Un profesional competente, 
más allá de estar calificado, es capaz de comprender una base conceptual que le hace posible ejercer su autonomía y creatividad en su vida laboral.

\section{Competencias de la rama de ingeniería}

La arquitectura e ingeniería es una rama del saber caracterizada por unos fundamentos comunes de formación matemático-técnica y una competencia profesional específica "la capacidad de proyectar y dirigir la ejecución de una obra" (Olds et al, 2004). Durante los últimos años, diversas organizaciones en los Estados Unidos (Academias Nacionales, agencias federales, organizaciones empresariales y asociaciones profesionales) han impulsado numerosos estudios que revelan la necesidad de nuevos paradigmas en la práctica, la investigación y la formación en la ingeniería que dirijan mejor las necesidades de la sociedad en el siglo XXI, en un mundo que cambia con rapidez (ABET, 2003, 2004; Duderstadt, 2008).

Hay que resaltar que en la mayoría de los países e instituciones, los trabajos sobre educación en la ingeniería coinciden en los puntos fundamentales (Olds et al, 2005; Martín et al, 2008). Se debe prestar atención a la praxis (la gestión de la calidad, habilidades de comunicación, trabajo en equipo, ética profesional, etc.) y a la técnica (diseño, creatividad, métodos de resolución de problemas, etc.), lo que combinado con la educación científica (fundamentos), que predomina en la actualidad en la mayoría de las ingenierías, y una más adecuada conexión entre la tecnología y la sociedad, aumentaría la eficacia de las actividades del ingeniero en el cumplimiento de su propósito: producir cosas útiles en beneficio de la humanidad (El-Raghy, 1999; Callaos, 2008).

El concepto actual de ingeniería se apoya en tres pilares básicos: la ciencia, la técnica y la ejecución o práctica. El desarrollo de los conocimientos científicos, la innovación tecnológica y las actividades de gestión o procedimientos técnicos de diseño organizativo son tres dimensiones importantes en la concepción de la ingeniería como un todo. Las características básicas del "ingeniero global" es que son técnicamente hábiles, cultos y con conocimientos generales. Son aquellos que presentan un espíritu empresarial y que son innovadores y aprendices toda la vida; ingenieros que comprenden los mercados mundiales, que conocen la manera de trasladar la innovación tecnológica a productos y servicios comercialmente viables; e ingenieros que, profesionalmente, están ágiles, flexibles y móviles (Continental, 2006). En consecuencia, los cambios más importantes que deben introducirse en los estudios de ingeniería tienen que ver con las competencias (praxis) más que con los contenidos (ciencia), si queremos satisfacer las necesidades generadas por el fenómeno de la globalización.

La dificultad de educar en competencias se ha puesto de manifiesto en diferentes trabajos (Walther y Radcliffe, 2007). La metodología de enseñanza y evaluación de competencias requiere un gran esfuerzo de coordinación por parte de los profesores. Una competencia no se puede enseñar sólo a través de una materia de los estudios de grado, sino que se debe desarrollar de forma gradual a lo largo de las diferentes etapas de formación de los graduados. La guía de competencias profesionales de la Federación Europea de Asociaciones Nacionales de Ingenieros (FEANI, 2005) que agrupa cerca de 2.000 .000 de ingenieros a través de las Asociaciones de 27 países europeos, recoge una serie de competencias (genéricas, específicas y transversales) que los ingenieros conscientes de sus responsabilidades profesionales deben esforzarse por lograr. La red temática University Studies of Agricultural Engineering in Europe (USAEE) siguiendo las recomendaciones de la FEANI, también establece una serie de habilidades para un graduado en ingeniería de 3 años (USAEE, 2006).

De acuerdo con este último documento, un profesional de la ingeniería debe participar en trabajos interdisciplinarios y, con frecuencia, de ámbito internacional. Por ello, sus competencias no deben limitarse a las requeridas por empresas o asociaciones europeas. Es muy importante establecer nexos comunes entre instituciones de educación superior con Iberoamérica y, en este sentido, el proyecto Tuning-América Latina (González et al, 2004) ha desarrollado un listado de 27 competencias genéricas con 22 puntos convergentes con el listado europeo (Tuning América Latina, 2007). Estos puntos identificados son necesarios para tender los puentes destinados al reconocimiento de las titulaciones en la región y con otras regiones del planeta. 
En el informe final del Seminario Internacional "Currículo Universitario Basado en Competencias" (Centro InterUniversitario de Desarrollo, 2005) se recoge la opinión de diferentes autoridades académicas de Iberoamérica. Entre ellas destacamos algunas ideas sobre la formación de los ingenieros: i) Entre las capacidades generales más importantes que debe tener un ingeniero está la capacidad para actuar en la sociedad de la información, participar de un mundo globalizado, el espíritu crítico, creativo y emprendedor que le sirva para generar empleo y para desarrollar todas las dimensiones del ser humano incluyendo la formación de valores; ii) Un ingeniero necesita desarrollar: el pensamiento lógico, la capacidad de investigar, el pensamiento estratégico, la comunicación verbal, el dominio del idioma inglés, la creatividad, la empatía y la conducta ética.

El Instituto Tecnológico de Santo Domingo (INTEC) nomina las competencias del perfil de un ingeniero entre las que se destaca la rectitud moral, la responsabilidad social, la ética, la creatividad y en que se destaca el acervo cultural, la capacidad de comunicación, el compromiso y la capacidad de trabajo en equipo, la capacidad para enfrentar y resolver problemas, la capacidad para integrar conocimientos y para participar en discusiones sobre el mundo actual, el manejo informático, el compromiso con el medio ambiente, la capacidad para proteger la identidad cultural propia y para respetar otras, y la capacidad de tomar decisiones con libertad y autonomía. En el informe final del Proyecto Tuning América Latina, 2007 se incluye un estudio específico del campo de la ingeniería civil, donde se definen además 19 competencias específicas para esta área de conocimiento, así como el desarrollo de algunas propuestas metodológicas para obtener resultados en dos de dichas competencias.

A partir de la publicación del Real Decreto (RD) 1393/2007, de 29 de octubre, (BOE 30/10/2007) por el que se establece la ordenación de las enseñanzas universitarias oficiales, las universidades españolas deben conseguir la progresiva armonización de los sistemas universitarios, exigida por el proceso de construcción del EEES. Esta normativa recoge que se garantizarán, como mínimo, las siguientes competencias básicas en el caso del Grado, y aquellas otras que figuren en el Marco Español de Cualificaciones para la Educación Superior (BOE 19/julio/2007):

1.- Que los estudiantes hayan demostrado poseer y comprender conocimientos en su área de estudio.

2.- Que los estudiantes sepan aplicar sus conocimientos a su trabajo o vocación de una forma profesional y posean las competencias que suelen demostrarse por medio de la elaboración y defensa de argumentos y la resolución de problemas dentro de su área de estudio.

3.- Que los estudiantes tengan la capacidad de reunir e interpretar datos relevantes (normalmente dentro de su área de estudio) para emitir juicios que incluyan una reflexión sobre temas relevantes de índole social, científica y ética.

4.- Que los estudiantes puedan transmitir información, ideas, problemas y soluciones a un público tanto especializado como no especializado.

5.- Que los estudiantes hayan desarrollado aquellas habilidades de aprendizaje necesarias para emprender estudios posteriores con un alto grado de autonomía.

\section{La Ingeniería Forestal}

En el caso del Ingeniero Forestal, predomina el ámbito sobre la especialidad; ya que tiene su acción profesional en el monte, concepto difícil de definir ya que abarca un extenso territorio y que, con delimitación negativa, viene a comprender los espacios exteriores a la habitación humana y no dedicados a cultivos con rotación anual de trabajo para la obtención de productos ni a industrias o comunicaciones. Esto corresponde a todo lo que supone una Naturaleza menos transformada por la actividad humana y que es origen de recursos y bienes absolutamente imprescindibles para el hombre actual; unos perfectamente medibles como maderas, pastos, resinas, frutos y otros de difícil cuantificación como la expresión del ocio o recreo, la calidad del paisaje y medio ambiente, los equilibrios ciudad-campo y dentro de éste el agro-silvo-pastoral, la función protectora, etc. (Grande et al, 2008). 
El European Forest Sector Outlook Studies (United Nations, FAO, 2005) señala que la demanda de productos forestales continuará creciendo la próxima década; aunque el crecimiento, en general, será inferior que en el pasado. En el sector forestal se prevé un aumento del uso de Sistemas de Información Geográfica y teledetección. La innovación tecnológica se centrará en las energías renovables, la selvicultura de precisión y la localización y control automatizado de incendios. En cuanto a la praxis, se demandará más protección del medioambiente, en general; y por tanto, se requerirán expertos en calidad de agua y de suelos. Además aumenta la demanda en expertos sociales en relación con los usos recreativos del monte (Bureau of Labor Statistics, 2009).

La encuesta laboral y de calidad de enseñanza en Ingeniería Forestal (Colegio Profesional de Ingenieros de Montes, 2002) muestra una ocupación mayoritaria en la gestión del medio natural $(12,34 \%)$, los trabajos típicamente forestales $(9,94 \%)$ y la restauración hidrológica forestal (9\%).para los titulados españoles. En muchos países europeos la ingeniería forestal está unida a la ingeniería agraria y no hay grandes diferencias de formación entre ambas, como es el caso de universidades de Austria o Suecia. Los trabajos sobre competencias de Ingenieros Agrónomos están más desarrollados que en el caso del Ingeniero Forestal (Brumm et al, 2006; USAEE, 2006). El conjunto de universidades españolas donde se imparten titulaciones relacionadas con la ingeniería agroforestal han llevado a cabo un trabajo exhaustivo, debatiendo y valorando distintas opciones, con el objetivo de alcanzar un modelo final consensuado (González, 2008). El proyecto presentado como "libro blanco" recoge numerosos aspectos fundamentales en el diseño de un modelo de título de grado; entre otros: análisis de los estudios correspondientes o afines en Europa, características de la titulación europea seleccionada, estudios de inserción laboral de los titulados durante el último quinquenio, y perfiles y competencias profesionales.

Del libro blanco sobre los "Estudios de Grado en Ingenierías Agrarias e Ingenierías Forestales" (ANECA, 2005) hay que destacar el análisis sobre perfiles profesionales para el caso de Ingenieros Forestales de ciclo corto (3 años) y largo (6 años), que resultaron: a) Proyectos y consultorías, 18,3\%; b) Selvicultura, ordenación de montes y pascicultura, 14,5\%; c) Protección e incendios forestales, 11,5\%; y d) Jardinería y paisajismo, $4,5 \%$

En cuanto a las competencias que deberían adquirirse se recoge la opinión de egresados y empleadores. Las competencias generales que los egresados consideran más necesarias para su profesión, por orden de importancia resultaron:

1. Capacidad de organización y planificación

2. Resolución de problemas

3. Capacidad de aplicar los conocimientos en la práctica

4. Capacidad de análisis y síntesis

5. Toma de decisiones

En cuanto a las competencias valoradas por los empleadores se agrupan en transversales, específicas y profesionales. Las competencias transversales menos valoradas actualmente son: el trabajo en contexto internacional y las herramientas que necesita (incluido el idioma). Las competencias actuales más valoradas son: capacidad de organización y planificación, resolución de problemas, trabajo en equipo y capacidad de aplicar los conocimientos a la práctica. Las competencias específicas más valoradas actualmente son: los conocimientos teóricos básicos y la gestión y control de la calidad. La competencia profesional más valorada actualmente es la elaboración y ejecución de estudios técnicos, y la menos valorada es la elaboración y ejecución de trabajos de I+D.

\section{Metodología}

La metodología empleada es la toma de decisiones basada en el juicio de expertos, que se obtiene mediante una de las técnicas de investigación cualitativa más usada en la actualidad: las técnicas grupales (Colectivo IOÉ, 2010). Las técnicas grupales se han utilizado especialmente en época de cambio y como una manera de conocer el comportamiento del ciudadano ante las novedades: sus conocimientos y actitudes, sus opiniones, expectativas, conflictos, su capacidad 
para asumir innovaciones, etc. (Amezcua, 2003). Una de las técnicas grupales empleadas en la investigación cualitativa es el grupo de discusión. En términos generales, el grupo de discusión consiste en una conversación planeada con un conjunto de personas, las cuales conocen el tema de discusión y se les hace una entrevista con un temario. Los temas están previamente definidos, pero son flexibles. El ambiente de discusión dentro de los grupos es permisivo y no-directivo (Vargas e Ibáñez, 2008) La evaluación mediante grupos de discusión está diseñada para obtener información en un área definida de interés particular. Las personas que son entrevistadas en los grupos deben ser individuos expertos en determinado tema o poseer mucha experiencia en el área. Una corriente de pensamiento considera relevante trabajar con grupos de personas que se conozcan entre sí o que correspondan a grupos ya constituidos llamados grupos naturales. Los grupos naturales deben seleccionarse en contextos cercanos o propios de los grupos de estudio (Lunt y Livingstone, 1996). Los grupos de discusión naturales se consideran los más apropiados para el estudio de las cogniciones sociales, como son las competencias curriculares. Además, trabajar con grupos naturales asegura que el marco social de interacción, así como las reglas básicas de conversación y de atribución de sentido, se compartan por los grupos (Pérez-Sánchez \& Víquez-Calderón, 2010).

En este trabajo se emplearon dos grupos naturales. El primero formado por algunos miembros del Grupo de Innovación Educativa en Técnicas Cuantitativas para la Ingeniería Medioambiental de la UPM en España y el segundo grupo formado por expertos en el área de Ingeniería Agroforestal europea, asistentes a la sesión de educación en la reunión bianual de EurAgeng del 2008. El primer grupo lo formaron 8 expertos con las siguientes características: i) Nacionalidad española; ii) Titulación de Doctor en el área de Ingeniería Agroforestal; iii) Amplia experiencia docente en el área (más de 5 años de antigüedad y más de dos materias diferentes); iv) Variada experiencia en la gestión institucional (liderazgo de grupos de trabajo, labores de gestión docente, dirección de ordenación académica, etc.); y v) Heterogeneidad de sexos y rango de 35 a 65 años de edad. El segundo grupo, de 10 personas, más heterogéneo en nacionalidad, edad y experiencia, compartía con el anterior la titulación de sus miembros.

\section{RESULTADOS}

Las competencias se deben desarrollar de forma progresiva. Éstas se adquieren en diferentes etapas del programa, por lo que en el diseño de éste se debe incluir en qué unidades concretas (asignaturas o materias) se desarrollará la formación en una particular competencia. La nueva estructura de las titulaciones de grado debe interconectar las materias, procurando que no sean compartimentos estancos, como son actualmente. Una forma clara es que un grupo de materias formen en una competencia o grupo de competencias. Con todos los antecedentes anteriores se ha elaborado una propuesta de competencias que deberían adquirirse en el ciclo formativo de grado en la titulación de Ingeniería Forestal. Las competencias se han agrupado en genéricas y específicas. Mientras que existe un gran consenso en la mayoría de las competencias genéricas, como se ha visto en los antecedentes, apenas se han desarrollado las competencias específicas del Ingeniero Forestal.

\section{Competencias Genéricas}

G1. Responsabilidad moral y profesional.

G2. Juicio crítico y creatividad.

G3. Comunicarse efectivamente.

G4. Analizar, plantear y solucionar problemas reales en ingeniería.

G5. Relacionarse y trabajar en equipo.

G6. Liderar equipos y tomar decisiones con autonomía.

G7. Fluidez para expresarse en diferentes idiomas.

G8. Fomentar el desarrollo propio y la mejora continua, adaptándose al entorno.

G9. Reconocer la incertidumbre del conocimiento y actitud innovadora.

G10. Formar a otros profesionales. 


\section{Competencias Específicas}

Los estudios realizados sobre estas competencias en el campo de la ingeniería forestal están menos desarrollados que los anteriores. Se han considerado dos grandes grupos: el de competencias específicas de la ingeniería y las específicas de la rama forestal. La propuesta siguiente es el resultado de acuerdos entre los miembros del Grupo de Innovación Educativa en Técnicas Cuantitativas para la Ingeniería Ambiental:

Competencias específicas de la ingeniería (I):

1. Obtener e interpretar información de campo.

2. Utilizar las nuevas tecnologías de la información.

3. Identificar, evaluar e implementar las tecnologías más apropiadas para su contexto.

4. Crear, innovar y emprender para contribuir al desarrollo tecnológico.

5. Evaluar el impacto ambiental y social de su actividad.

6. Administrar recursos materiales y humanos, así como diferentes equipos.

Competencias específicas de la rama forestal $(F)$ :

1. Aplicar conocimientos de las ciencias básicas de la ingeniería forestal.

2. Concebir, analizar, proyectar y diseñar obras de ingeniería forestal.

3. Planificar y programar obras y servicios propios de la ingeniería forestal.

4. Realizar, supervisar e inspeccionar obras de ingeniería forestal.

5. Modelizar y simular sistemas naturales y procesos de ingeniería forestal.

6. Tomar decisiones y gestionar los recursos naturales.

7. Encontrar soluciones que contribuyan a la sostenibilidad de los recursos naturales.

8. Interactuar con grupos multidisciplinares para dar soluciones integrales al medio ambiente.

Las materias necesarias para la formación del Ingeniero Forestal se agrupan en tres bloques: materias básicas, fundamentos de la Ingeniería y fundamentos de las Ciencias Agroforestales. Hay materias pertenecientes a cada bloque que pueden basarse en alguno o varios de los tres pilares de la ingeniería: la ciencia, la técnica y la ejecución o práctica. A continuación, se representan, mediante diagramas de conjuntos (figura 1), las bases de la ingeniería, con el fin de mostrar un esquema del desarrollo de las competencias en relación con estas bases.
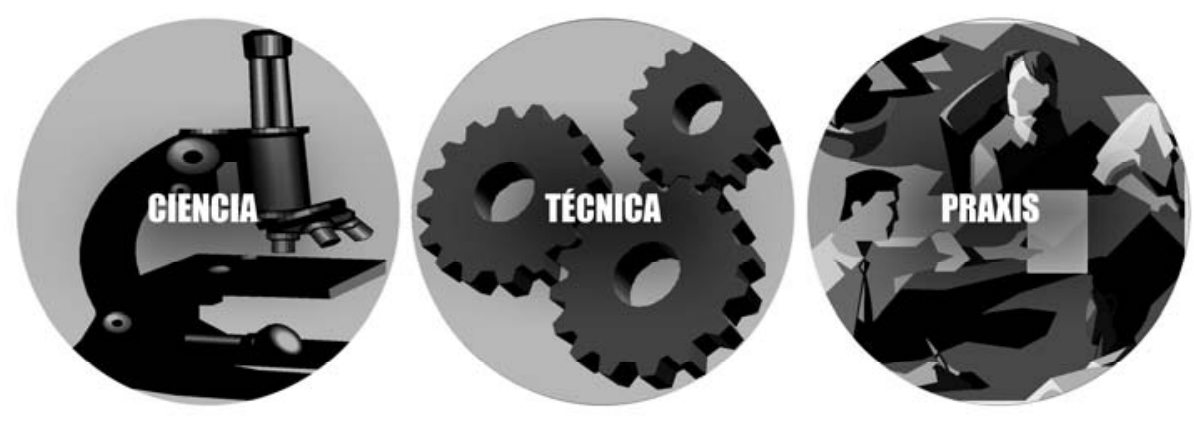

Fig. 1. Representación gráfica de las bases de la Ingeniería.

Los diagramas de Euler-Venn permiten visualizar cómo se desarrollaría la formación en competencias dependiendo del bloque de materias. Los elementos de los conjuntos son las competencias. Las competencias generales se denominan con la letra G, las específicas de la ingeniería con I y las específicas de la rama forestal con F. 
La importancia de la formación científica en materias básicas y su relación con las competencias generales se representa en la figura 2.

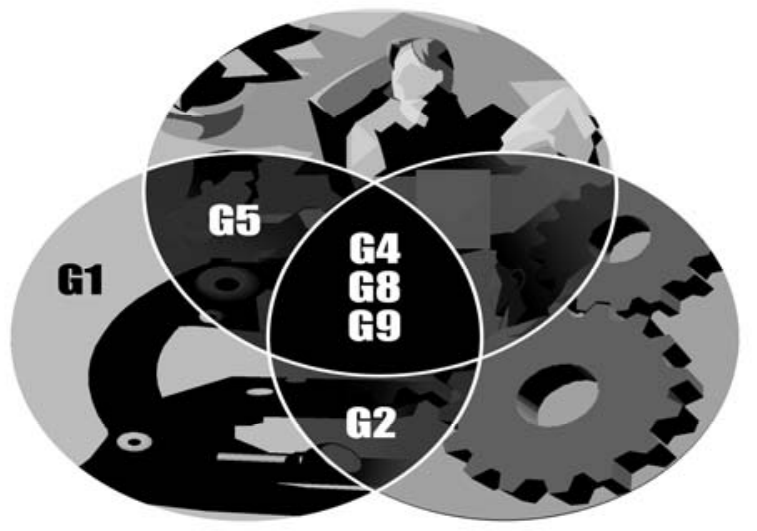

Fig. 2. Competencias en materias básicas.

La importancia de la formación práctica y científico-técnica en las materias del bloque fundamentos de la ingeniería y su relación con las competencias específicas de la ingeniería se representa en la figura 3.

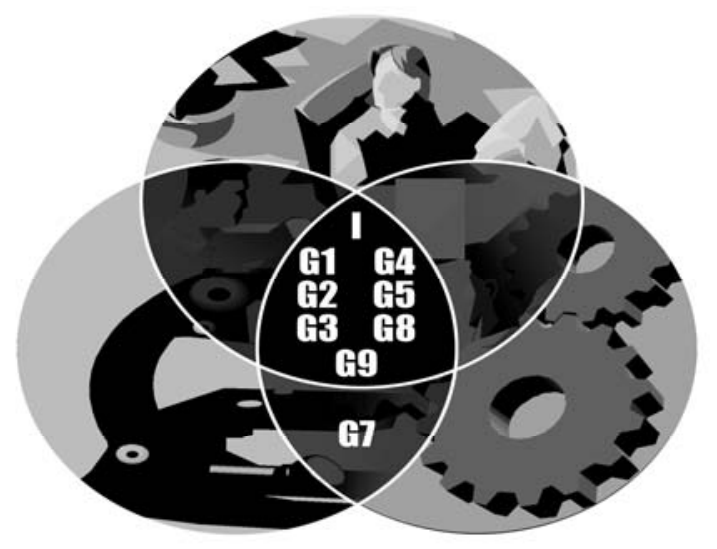

Fig. 3. Competencias en fundamentos de la ingeniería.

La figura 4 muestra la importancia de la formación práctica en las materias del bloque fundamentos de las ciencias agroforestales y su relación con las competencias específicas de la ingeniería forestal.

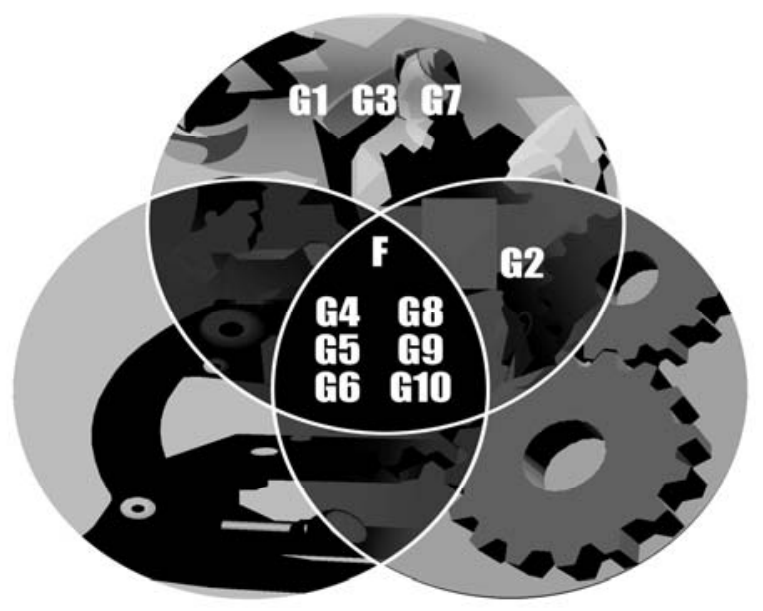

Fig. 4. Competencias en fundamentos de las ciencias agroforestales. 


\section{CONCLUSIONES}

La formación en competencias es uno de los fundamentos del EEES. Los trabajos previos muestran que el mayor consenso en la comunidad de profesionales y académicos de todo el mundo se da en las competencias que deben tener los egresados.

La formación en competencias para un graduado es compleja y requiere un esfuerzo conjunto de profesores de diferentes materias y bloques de materias.

La formación de un ingeniero debe tener tres apoyos básicos: la ciencia, la técnica y la praxis. Las competencias de los profesionales en ingeniería forestal deben ser genéricas, específicas de la ingeniería y específicas de su especialidad.

En las materias básicas para la formación del Ingeniero Forestal destaca la importancia de la formación científica y su relación con las competencias generales. En las materias relacionadas con los fundamentos de la ingeniería son más importantes las componentes práctica y científicotécnica y están relacionadas con las competencias específicas de la ingeniería. En cuanto a las materias que se ocupan de los fundamentos de las ciencias agroforestales, se puede resaltar la importancia de la formación práctica y su relación con las competencias específicas de la ingeniería forestal.

\section{REFERENCIAS}

ABET, Accrediting Board for Engineering and Technology, Criteria for Accrediting Engineering Programs, Inc. Baltimore, Maryland, (en línea), 2003, 2004, www.abet.org/criteria_eac.html Acceso: 15 de mayo (2010).

Amezcua, M., La entrevista en grupo. Características, tipos y utilidades en investigación cualitativa. Enfermería Clínica, 13 (2), 112 - 117 (2003).

ANECA Libro Blanco. Estudios de Grado en Ingenierías Agrarias e Ingenierías Forestales (en línea), 2005. http://www.aneca.es/activin/docs/libroblanco_agrarias_forestales_def.pdf. Acceso: 20 de julio (2008).

Ayuga, E., La formación del Ingeniero Iberoamericano, en Convergencia Europea en la UPM: Ingenierías Agroforestales y Ciencias Ambientales, editores Ayuga \& González pp. 21-30. Fundación Conde del Valle de Salazar, ETSIM (UPM), Madrid (2008).

BBVA. Estudio Fundación BBVA: Internet en España (en línea), 2008. www.fbbva.es/ LFU/dat/Estudio_Internet_2008.pdf. Acceso: 22 de julio (2008).

BOE 19/julio/2007: REAL DECRETO 900/2007, de 6 de julio, por el que se crea el Comité para la definición del Marco Español de Cualificaciones para la Educación Superior.

BOE 30/octubre/2007: REAL DECRETO 1393/2007, de 29 de octubre por el que se establece la ordenación de las enseñanzas universitarias oficiales.

Brumm, T. J. y otros tres autores, Competency-based Outcomes Assessment for Agricultural Engineering Programs, International Journal of Engineering Education, 22(6) 1163-1172 (2006).

Bureau of Labor Statistics, U.S. Department of Labor, Occupational Outlook Handbook, 2008-09 Edition, Conservation Scientists and Foresters (2009) http://www.bls.gov/oco/ocos048.htm Acceso: 5 de febrero (2009).

Callaos, N., The Essence of Engineering and Meta-Engineering: A Work in Progress (en línea), $2008 \mathrm{http} / / / \mathrm{www}$.iiis.org/Nagib-Callaos/Engineering-and-Meta-Engineering/ Acceso: 5 de febrero (2009).

Centro InterUniversitario de Desarrollo. Seminario Internacional, Currículo Universitario Basado en Competencias, Universidad del Norte, Barranquilla, Colombia (2005). 
Colectivo IOÉ., ¿Para qué sirve el grupo de discusión? Una revisión crítica del uso de técnicas grupales en los estudios sobre migraciones. EMPIRIA. Revista de Metodología de Ciencias Sociales, No 19, 73-99 (2010).

Colegio profesional de Ingenieros de Montes, I Informe de resultados de la Encuesta Laboral y de Calidad de la Enseñanza en Ingeniería de Montes, Montes (2002).

Committee on the Offshoring of Engineering. The Offshoring of Engineering: Facts, Unknowns, and Potential Implications. The National Academy Press, 125-230 (2008).

Continental A.G., In Search of Global Engineering Excellence: Educating the Next Generation of Engineers for the Global Workplace. Hanover, Germany, Continental AG (2006) http://www.contionline.com

Deakin, R., Key Competencies for Education in a European Context: narratives of accountability or care, European Educational Research Journal: 7(3), 311-318 (2008).

Duderstadt, J.J., Engineering for a Changing World: A Roadmap to the Future of Engineering Practice, Research, and Education, in Millennium Project, University of Michigan (2008). http://milproj.ummu.umich.edu/publications/EngFlex_report/download/EngFlex\%20Report.pdf

FEANI. Guide To The Feani Register Eur Ing (2005). http://www.feani.org/Guide\%20to\%20 he\%20Feani\% ORegister.doc. Acceso: 5 de febrero (2009).

El-Raghy, S., Quality Engineering Education: Student Skills and Experiences. Global Journal of Engineering Education: 3 (1), 25-29 (1999).

Fredriksson, U., Changes of Education Policies within the European Union in the Light of Globalisation, European Educational Research Journal, 2(4), 522-546 (2003).

González, C., La Agencia Nacional de Evaluación de la Calidad y Acreditación (ANECA) y las titulaciones de Grado Agroforestales, en Convergencia Europea en la UPM: Ingenierías Agroforestales y Ciencias Ambientales, Ayuga \& González (editoras), pp 203-230. Fundación Conde del Valle de Salazar, ETSIM (UPM), Madrid (2008).

González, J.; R. Wagenaar y P. Beneitone, Tuning-América Latina: un proyecto de las universidades, Revista Iberoamericana de Educación: 35, 151-164 (2004).

Grande, M.A.; González, C., Ayuga, E., Propuesta curricular del GIETECIM para la titulación de grado en Ingeniería de Montes y Forestal, en Convergencia Europea en la UPM: Ingenierías Agroforestales y Ciencias Ambientales, Ayuga \& González (editoras), pp 241-259. Fundación Conde del Valle de Salazar, ETSIM (UPM), Madrid (2008).

Kallioinen, O., Defining and Comparing Generic Competences in Higher Education, European Educational Research Journal, 9(1), 56-68 (2010).

Korsunsky, A. M. Globalization, Networking, and Engineering Technologies. Proceedings of the IMECS 2010 March 17-19 2010 in Hong Kong. Vol III pp. 5 (en línea) http://www.iaeng.org/ ublication/IMECS2010/IMECS2010_pp1500-1504.pdf. Acceso 15 de mayo (2010).

Lunt, P. \& Livingstone, S., Rethinking the focus groups in media and communication research. Journal of Communication, 46, 79-98 (1996).

MacLeod, I. A., The education of innovative engineers. Engineering, Construction and Architectural Management: 17(1), 21-34 (2010).

Mardam-Bey, O.; Sanjay y S. Saran, Impact of Globalization on Engineering Education in Developing Countries, Arab Research Institute in Sciences \& Engineering Vol. 4 No. 2 (2008) 99102. http://www.arabrise.org/articles/A040205M.pdf 
Martín, S.; E. Ayuga y C. González, Globalización de las enseñanzas de ingeniería, en Convergencia Europea en la UPM: Ingenierías Agroforestales y Ciencias Ambientales, editores Ayuga \& González, pp 7-19. Fundación Conde del Valle de Salazar, ETSIM (UPM), Madrid (2008).

McEvoy, G. M. y otros cinco autores A, Competency-Based Model for Developing Human Resource Professionals, Journal of Management Education: 29(3), 383-402 (2005).

Miguel Díaz, M. de, Adaptación de los Planes de Estudios al Proceso de Convergencia Europea. (en línea), 2004. Proyecto EA2004-0024. Programa de Estudios y Análisis destinado a la Mejora de la calidad de la Enseñanza y de la Actividad del Profesorado Universitario. Dirección General de Universidades, Ministerio de Educación y Ciencia, Madrid,. http://www.ub.es/eees/documents/dfes/ ec/mec_2004.pdf. Acceso: 20 de mayo (2010).

Miguel Díaz, M. de (coord.), Metodologías de enseñanza y aprendizaje para el desarrollo de competencias. Orientaciones para el profesorado universitario ante el espacio europeo de educación superior. Madrid: Alianza Editorial. (2006).

Olds, B. M. y R. E. Miller, The Effect of a First-Year Integrated Engineering Curriculum on Graduation Rates and Student Satisfaction: A Eongitudinal Study, Journal of Engineering Education: 93(1), 23-35 (2004).

Olds, B. M.; B. M. Moskal, y R. L. Miller, Assessment in Engineering Education: Evolution, Approaches and Future Collaborations. Journal of Engineering Education, 94(1), 13-25 (2005).

Pérez-Sánchez, R. \& Víquez-Calderón, D. Los grupos de discusión como metodología adecuada para estudiar las cogniciones sociales. Actualidades en Psicología. 23-24, 2009-2010, 87-101 (2010).

Tomisawa Osamu, K. G. Entrepreneur Engineering-A New Concept of Engineering Education. Eizo Joho Media Gakkai Gijutsu Hokoku: 30(26), 33-38 (2006).

Tuning América Latina. Informe Final del Proyecto Tuning América Latina: Reflexiones y perspectivas de la Educación Superior en América Latina (2007), http://tuning.unideusto.org/tuningal/

index.php?option=com_docmanltemid=191\&task=view_category\&catid=22\&order=dmdate_publish ed\&ascdesc=DESC Acceso: 5 de febrero (2009).

Tuning Project. Reference Points for the Design and Delivery of Degree Programmes in European Studies. Universidad de Deusto, Bilbao, (2008).

Tuning Project. Tuning Educational Structures in Europe II. La contribución de las universidades al proceso de Bolonia. Julia González Editora. Universidad de Deusto, Bilbao, (2006).

Tuning Project. Tuning Educational Structures in Europe. Universidad de Deusto, Bilbao, (2003).

United Nations Economic Commission for Europe/Food and Agriculture Organization of the United Nations.(FAO. European Forest Sector Outlook Studies. Ed. United Nations, Ginebra (2005).

Universia. Historia del Espacio Europeo de Educación Superior. http://eees.universia.es/historia/ index.htm. Acceso: 5 de febrero (2009).

USAEE. Proposed USAEE Core Curriculum (2006), http://www.eurageng.net/files/usaeecorecurriculum.pdf. Acceso: 5 de febrero (2009).

Vargas Flores, J. J. \& Ibáñez Reyes, E. J. Grupos focales en la evaluación de materiales didácticos para la educación superior. Revista Electrónica de Psicología Iztacala, Vol. 11 No. 3, 68-82 (2008).

Walther, J. y D.F. Radcliffe, The competence dilemma in engineering education: Moving beyond simple graduate attribute mapping, Australasian Journal of Engineering Education, 13(1), 41-51 (2007). 